\title{
Indescribable cardinals without diamonds
}

\author{
Kai Hauser \\ Department of Mathematics, University of Michigan, Ann Arbor, MI 48109, USA
}

Received April 8, 1991/in revised form February 20, 1992

Summary. We show that for $m, n \geqq 1$ the existence of a $\Pi_{n}^{m}$ indescribable cardinal is equiconsistent with the failure of the combinatorial principle $\diamond_{\kappa, R e g n \kappa}$ at a $\Pi_{n}^{m}$ indescribable cardinal $\kappa$ together with the Generalized Continuum Hypothesis.

\section{Introduction}

Recall the combinatorial principle

$$
\begin{aligned}
& \diamond_{\kappa, E}: \text { There is a sequence }\left\langle S_{\alpha}: \alpha \in E\right\rangle \text { such that } \forall \alpha \in E\left(S_{\alpha} \subseteq \alpha\right) \\
& \text { and } \forall S \subseteq \kappa\left\{\alpha \in E: S_{\alpha}=S \cap \alpha\right\} \text { is stationary in } \kappa,
\end{aligned}
$$

where $E$ is a stationary subset of some cardinal $\kappa$. Jensen (cf. [D2]) showed that if $\kappa$ is regular and uncountable and $V=L$ then $\diamond_{\kappa, E}$ holds. Following his work, a number of applications of this principle and its modifications have been developed which are wide ranging and not restricted to set theory (cf. [D1]). Jensen had also discovered that various large cardinals carry diamond sequences with them; for example if $\kappa$ is ineffable or even if $\kappa$ is subtle (see [D2] and [KM] for the definitions of these concepts) then $\diamond_{\kappa, \text { Regn }}$ holds (where Reg denotes the class of all regular cardinals). It has been asked by several people whether cardinals below the least subtle cardinal carry diamond sequences. In this paper we show that it is consistent that $\diamond_{\kappa, \text { Regn }}$ fails at a $\Pi_{n}^{m}(m \geqq 1, n \geqq 1)$ indescribable cardinal $\kappa$.

Assuming that $\kappa$ is $\Pi_{n}^{m}$ indescribable, we shall work in $L$ and define an iteration $P$ of length $\kappa+1$ which at stage $\lambda \leqq \kappa$ kills off all candidates for $\diamond_{\lambda, \text { Regn } \lambda}$ sequences. In order to show that $P$ preserves the $\Pi_{n}^{m}$ indescribability of $\kappa$ we use the elementary embedding characterization of $\Pi_{n}^{m}$ indescribability in [H1] and master condition arguments. $P$ will preserve all cofinalities and not add too many sets. Thus we obtain

Current address: Department of Mathematics, University of California, Berkeley, CA 94720, USA 
Theorem. ( $m \geqq 1, n \geqq 1)$

$\operatorname{CON}\left(Z F C+\exists \Pi_{n}^{m}\right.$ indescribable $) \Leftrightarrow$

$\operatorname{CON}\left(Z F C+\exists \kappa\left(\kappa\right.\right.$ is $\Pi_{n}^{m}$ indescribable $\left.\left.\wedge \neg \diamond_{\kappa, \text { Regn } \kappa}\right)+G C H\right)$.

This generalizes a result of Woodin (cf. [CW]) who proved independently that $\neg \diamond_{\kappa, R e g \cap \kappa}$ at a weakly compact cardinal together with $G C H$ is equiconsistent with the existence of a weakly compact cardinal.

Aside from the usual set theoretic notation we shall also use the following symbols: $A d d(I, \kappa)$ denotes the $<\kappa$ support product indexed by $I$ of copies of the forcing for adding a subset of $\kappa$ with conditions of size $<\kappa$. Thus conditions in $\operatorname{Add}(I, \kappa)$ are functions $f$ with domain $I$ such that $|\{i: f(i) \neq \emptyset\}|<\kappa$ and for $i \in I$ with $f(i) \neq \emptyset$ there is a $\beta<\kappa$ with $f(i): \beta \rightarrow\{0,1\}$. We shall frequently fail to distinguish between $f(i)$ and $\{\xi \in \operatorname{dom}(f(i)): f(i)(\xi)=1\}$. For $S \cong I,\left.f\right|^{\emptyset} S \in \operatorname{Add}(I, \kappa)$ is obtained from $f \in \operatorname{Add}(I, \kappa)$ by restricting its support to $S . \operatorname{Add}^{I}(S, \kappa) \overline{\overline{\mathscr{Q} f}}\left\{\left.f\right|^{\emptyset} S: f \in \operatorname{Add}(I, \kappa)\right\}$. A nice $\operatorname{Add}(I, \kappa)$ name for a subset of $\kappa$ is essentially a function with domain $\kappa$ and range contained in the set of all antichains of $\operatorname{Add}(I, \kappa)$, i.e., a nice $\operatorname{Add}(I, \kappa)$ name $\tau$ for a subset of $\kappa$ is of the form $\tau=\bigcup_{\alpha<\kappa}\left(\{\tilde{\alpha}) \times A_{\alpha}\right)$ where each $A_{\alpha}$ is an antichain in $\operatorname{Add}(I, \kappa)$. An enumeration $\left\langle\tau_{\zeta}: \zeta\left\langle\kappa^{+}\right\rangle\right.$of nice $\operatorname{Add}(I, \kappa)$ names for subsets of $\kappa$ is called complete if every nice $A d d(I, \kappa)$ name for a subset of $\kappa$ appears cofinally often in the enumeration (here we assume $|I|=\kappa^{+}$and $G C H$ ). If $M$ is a transitive model of $Z F^{-}$(some suitable fragment of $Z F C$ ) and $\alpha \in M$, then $(M)_{\alpha}$ denotes $M$ 's version of $V_{\alpha}$, i.e. $V_{\alpha} \cap M$. However we shall also use the notation $M \models " V_{\alpha} \models \Phi(A)$ ". $M$ is called $\Sigma_{n}^{m}(m \geqq 1, n \geqq 0)$ correct for $\kappa$ iff $M^{\left|V_{\kappa+m-2}\right|} \subseteq M$ (that is $M^{<\kappa} \subseteq M$ if $\left.m=1\right)$ and $M$ correctly computes the $\sum_{n}^{m}$ facts over $V_{\kappa}$ that hold in parameters from $(M)_{\kappa+m}$.

I would like to thank Professor $\mathrm{H}$. Woodin for helpful discussions on this topic. I am also indebted to Professor A. Blass for patiently listening to several versions of the proof (including some wrong ones) and for his suggestions for improvements to this paper.

\section{Definition of the iteration}

Before we give the definition of the iteration $P$ we present for a given cardinal $\lambda$ a poset $Q^{\lambda}$ which forces $\neg \diamond_{\lambda, \operatorname{Reg} \cap \lambda \cdot} Q^{\lambda}$ could be defined as an iteration of length $\lambda^{+}$ which kills off one-by-one all potential $\diamond_{\lambda, \operatorname{Reg} \cap \lambda}$ sequences. However, for our purposes it is more useful to think of $Q^{\lambda}$ as a concrete suborder of $\operatorname{Add}\left(\lambda^{+} \times 2, \lambda\right)$. For each $\alpha<\lambda^{+}, Q^{\lambda}$ will add at coordinate $\langle\alpha, 0\rangle$ a set $S_{\alpha} \subseteq \lambda$ and at coordinate $\langle\alpha, 1\rangle$ a club set $C_{\alpha} \subseteq \lambda$ such that the pair $\left(S_{\alpha}, C_{\alpha}\right)$ is a counterexample to a certain candidate (given by a name in the forcing up to stage $\alpha$ ) being a $\diamond_{\lambda, R e q \neg \lambda}$ sequence. The formal definition is as follows. Define by induction on $\alpha \leqq \lambda^{+}$a sequence $\left\langle Q_{\alpha}^{\lambda}: \alpha \leqq \lambda^{+}\right\rangle$such that each $Q_{\alpha}^{\lambda}$ is a suborder of $A d d^{\lambda^{+} \times 2}(\alpha \times 2, \lambda)$ and let $Q^{\lambda} \overline{\overline{D f}} Q_{\lambda^{+}}^{\lambda}$. Fix a complete enumeration of nice $\operatorname{Add}\left(\lambda^{+} \times 2, \lambda\right)$ names for subsets of $\lambda$, say $\left\langle\tau_{\zeta}: \zeta<\lambda^{+}\right\rangle$(assuming $\bar{G} C H$ ). Let $Q_{0}^{\lambda} \overline{\overline{\mathscr{Q} f}}$ the trivial poset. For a limit ordinal $\alpha \leqq \lambda^{+}$ we set $Q_{\alpha}^{\lambda} \mathscr{Q}_{f}\left\{f \in A d d^{\lambda^{++} \times 2}(\alpha \times 2, \lambda): \forall \beta<\left.\alpha f\right|^{\phi} \beta \in Q_{\beta}^{\lambda}\right\}$. If $Q_{\alpha}^{\lambda}$ has been defined for some $\alpha<\lambda^{+}$we let

$$
\begin{aligned}
& Q_{\alpha+1}^{\lambda} \mathscr{Q P f}\left\{f \in A d d^{\lambda+\times 2}((\alpha+1) \times 2, \lambda):\left.f\right|^{\emptyset}(\alpha \times 2) \in Q_{\alpha}^{\lambda} \wedge[f(\langle\alpha, 0\rangle)\right. \\
& \quad=f(\langle\alpha, 1\rangle)=\emptyset \vee[\exists \beta<\lambda[\operatorname{dom}(f(\langle\alpha, 0\rangle)=\operatorname{dom}(f(\langle\alpha, 1\rangle)) \\
& \quad=\beta+1 \wedge f(\langle\alpha, 1\rangle)(\beta)=1] \wedge\{\eta: f(\langle\alpha, 1\rangle)(\eta)=1\} \text { is closed in } \lambda
\end{aligned}
$$


$\left.\left.\left.\left.\lambda \wedge f\right|^{\emptyset}(\alpha \times 2) \|_{Q_{\alpha}^{\prime}} " \forall \zeta \in \operatorname{Reg} \cap\{\eta: f(\langle\alpha, 1\rangle)(\eta)=1\}\{\eta: f(\langle\alpha, 0\rangle)(\eta)=1\} \cap \zeta \neq\left(\hat{\tau}_{\alpha}\right)_{\zeta} "\right]\right]\right\}$,

where

$$
\hat{\tau}_{\alpha} \overline{\overline{D f}}\left\{\langle\eta, g\rangle: g \in Q_{\dot{\gamma}} \wedge \exists h\left(\langle\eta, h\rangle \in \tau_{\alpha} \wedge g \leqq h\right)\right\}
$$

and $\left(\hat{\tau}_{\alpha}\right)_{\zeta}$ denotes (in $V^{Q \hat{\alpha}}$ ) the $\zeta$-th set in the potential diamond sequence given by $\hat{\tau}_{\alpha \text {. }}$

Note that compatibility in $Q^{\lambda}$ agrees with compatibility in $\operatorname{Add}\left(\lambda^{+} \times 2, \lambda\right)$. Thus $Q^{\lambda}$ is $\lambda^{+}$c.c. Moreover, for each $\mu<\lambda\left\{f \in Q^{\lambda}: \forall \zeta \in \operatorname{supp}(f) \sup f(\langle\zeta, 1\rangle)>\mu\right\}$ is a dense $\mu$-closed suborder of $Q^{\lambda}$. Thus $Q^{\lambda}$ is $<\lambda$ Baire and $\| \overline{Q^{\lambda}} \neg \diamond_{\lambda \text {, Regn } \lambda \text {. }}$.

Strictly speaking the definition of $Q^{\lambda}$ contains the sequence $\left\langle\tau_{\zeta}: \zeta\left\langle\lambda^{+}\right\rangle\right.$as a parameter. However, the actual choice is irrelevant as we shall see in a moment. First we need to isolate a property of sets $S \subseteq \lambda^{+} \times 2$ which guarantees that for any condition $f \in Q^{\lambda}$ its "restriction" $\left.f\right|^{\emptyset} S$ to the coordinates in $S$ is still a condition.

Definition 1.1. Call a set $S \cong \lambda^{+} \times 2$ complete (for a given enumeration $\left(\tau_{\zeta}: \zeta<\lambda^{+}\right)$of nice $\operatorname{Add}\left(\lambda^{+} \times 2, \lambda\right)$ names for subsets of $\left.\lambda\right)$ if

$$
\forall \zeta<\lambda^{+}(\langle\zeta, 0\rangle \in S \Leftrightarrow\langle\zeta, 1\rangle \in S)
$$

and

$$
\forall\langle\zeta, i\rangle \in S \forall\langle\eta, f\rangle \in \tau_{\zeta}(\operatorname{supp}(f) \subseteq \zeta \times 2 \Rightarrow \operatorname{supp}(f) \subseteq S) .
$$

Definition 1.2. Let $\left(\tau_{\zeta}: \zeta<\lambda^{+}\right)$be a complete enumeration of nice $\operatorname{Add}\left(\lambda^{+} \times 2, \lambda\right)$ names for subsets of $\lambda$ and let $S \subseteq \lambda^{+} \times 2$. For $\alpha<\lambda^{+}$we define

$$
{ }^{\mathrm{s}} Q_{\zeta}^{\lambda}=\left\{f \in Q_{\zeta}^{\lambda}: \operatorname{supp}(f) \subseteq S\right\}
$$

and

$$
{ }^{s} Q^{\lambda}={ }^{s} Q_{\lambda^{+}}^{\lambda}
$$

and

$$
s_{\tilde{\tau}_{\zeta}}=\left\{\langle\eta, f\rangle: f \in^{s} Q_{\zeta}^{\lambda} \wedge \exists g\left(\langle\eta, g\rangle \in \tau_{\zeta} \wedge f \leqq g\right)\right\} .
$$

If it is clear from the context which $S$ we are referring to, we drop the superscript $S$ and write $\tilde{\tau}_{\zeta}$. These definitions correspond to definitions 2.8 and 2.9 of [H2]. We can now show

Lemma 1.3. Suppose $S \subseteq \lambda^{+} \times 2$ is a complete set of coordinates for an enumeration $\left\langle\tau_{\zeta}: \zeta<\lambda^{+}\right\rangle$. Then for each $\alpha \leqq \lambda^{+}$

$$
\left.\forall q \in Q_{\alpha}^{\lambda} \quad q\right|^{\oplus} S \in^{S} Q_{\alpha}^{\lambda}
$$

and

$$
{ }^{s} Q_{\alpha}^{\lambda} \text { is a complete suborder of } Q_{\alpha}^{\lambda} .
$$

Proof. This is a simpler version of the proof of Lemma 2.10 in [H2]. Obviously the first claim implies the second. To prove the former we proceed by induction on $\alpha$. The cases $\alpha=0$ or $\alpha$ a limit are immediate. If $\alpha$ is a successor, say $\alpha=\beta+1$ and $\langle\beta, 0\rangle,\langle\beta, 1\rangle \in S$ use the fact that for any $H$ which is $Q_{\beta}^{\lambda}$ generic, $\left(\hat{\tau}_{\beta}\right)^{\bar{H}}=\left(\tilde{\tau}_{\beta}\right)^{H_{\cap} s_{Q_{\beta}^{\lambda}} \text { by }}$ the completeness of $S$. 
Equipped with this result we obtain:

Lemma 1.4. Suppose $\left\langle\tau_{\zeta}: \zeta\left\langle\lambda^{+}\right\rangle\right.$and $\left\langle\bar{\tau}_{\zeta}: \zeta<\lambda^{+}\right\rangle$are two complete enumerations of nice $A d d\left(\lambda^{+} \times 2, \lambda\right)$ names for subsets of $\lambda$ and $\left\langle Q_{\zeta}^{\lambda}: \zeta<\lambda^{+}\right\rangle$and $\left\langle\bar{Q}_{\zeta}^{\lambda}: \zeta<\lambda^{+}\right\rangle$are the corresponding posets for killing $\diamond_{\lambda, \operatorname{Regn} \lambda} \cdot Q^{\lambda}$ and $\bar{Q}^{\lambda}$ are isomorphic.

Proof. The isomorphism of $Q^{\lambda}$ onto $\bar{Q}^{\lambda}$ will be induced by a bijection $e: \lambda^{+} \rightarrow \lambda^{+}$. The construction of $e$ is carried out in a back-and-forth way in $\lambda^{+}$steps, i.e., we define a sequence $\left\langle e_{\alpha}: \alpha<\lambda^{+}\right\rangle$by induction on $\alpha$ such that:

(1) $e_{\alpha}$ is a $1: 1$ function, $\left|e_{\alpha}\right| \leqq \lambda, e_{\alpha} \subseteq e_{\beta}$ for $\alpha<\beta, \alpha \subseteq \operatorname{dom} e_{\alpha} \subseteq \lambda^{+}, \alpha \subseteq \operatorname{rng} e_{\alpha} \subseteq \lambda^{+}$.

(2) $\left(\operatorname{dom} e_{\alpha}\right) \times 2$ is complete for $\left\langle\tau_{\zeta}: \zeta\left\langle\lambda^{+}\right\rangle\right.$, (rng $\left.e_{\alpha}\right) \times 2$ is complete for $\left\langle\bar{\tau}_{\zeta}: \zeta\left\langle\lambda^{+}\right\rangle\right.$, and $e_{\alpha}$ induces an isomorphism of $\left(\right.$ dom $\left.e_{\alpha}\right) \times 2_{Q} \lambda$ with $\left(\operatorname{rng} e_{\alpha}\right) \times 2_{{ }_{Q}} \lambda$.

Let $e_{0}=\emptyset$ and for $\alpha<\lambda^{+}$a limit let $e_{\lambda}=\bigcup_{\eta<\lambda} e_{\eta}$. If we arrive at a successor $\alpha$, say $\alpha=\beta+1$ and $\beta \notin$ dom $e_{\beta}$ let $\gamma>\sup$ rng $e_{\beta}$ be least such that $\bar{\tau}_{\gamma}=\left(\hat{\tau}_{\beta}\right)^{e_{\beta}}$ and define $e_{\alpha}(\beta)=\gamma$. If $\beta \notin \operatorname{rng} e_{\beta} \cup\{\gamma\}$ pick the least $\delta>\sup \left(\operatorname{dom} e_{\beta} \cup\{\beta\}\right)$ such that $\tau_{\delta}=\left(\hat{\bar{\tau}}_{\beta}\right)^{e^{-1}}{ }^{1}$ and let $e_{\alpha}(\delta)=\beta$. Here $\left(\hat{\tau}_{\beta}\right)^{e_{\beta}}$ and $\left(\hat{\bar{\tau}}_{\beta}\right)^{e_{\bar{\beta}}{ }^{1}}$ denote the shift of these terms under the corresponding induced embeddings. If $\beta \in \operatorname{dom} e_{\beta}$ or $\beta$ is in the range of the intermediate function we skip the corresponding clauses in the definition of $e_{\alpha}$. The definition works since the sequences of terms are complete. Let $e=\bigcup_{\alpha<\lambda^{+}} e_{\alpha}$. More details can be extracted from the proof of Lemma 2.11 in [H2].

In order to prove the non trivial direction of the Theorem we can assume $V=L$ (since indescribability relativizes down to $L$ ) and $\kappa$ is $\Pi_{n}^{m}$ indescribable ( $m \geqq 1, n \geqq 1$ ). Notice that the failure of $\diamond_{k, \text { Regn }}$ is expressible by a $\Pi_{2}^{1}$ sentence over $\left\langle V_{\kappa}, \varepsilon\right\rangle$. Hence simply forcing with $Q^{\kappa}$ will not do the job if we want $\kappa$ to be (at least) $\Pi_{2}^{1}$ indescribable in the final model. (If $m=n=1$ note that in $L[G]$, where $G$ is $Q^{\kappa}$ generic over $V=L$, the $\Pi_{1}^{1}$ statement (over $\left\langle V_{\kappa}, \varepsilon\right\rangle$ ) " $X \notin L$ " will not reflect below $\kappa$ for any "new" $X \subseteq \kappa$ since $Q^{\kappa}$ is $<\kappa$ Baire). Our forcing notion will be an iteration $\left\langle P_{\alpha}: \alpha \leqq \kappa+1\right\rangle$ which not only kills $\diamond_{\kappa, \text { Reg } \cap \kappa}$ but also $\diamond_{\lambda, \text { Regn } \lambda}$ for "many" $\lambda<\kappa$. Here is the official defition: $P_{0}$ is the trivial poset. If $\lambda$ is a limit ordinal $\leqq \kappa$ then $P_{\lambda}=\lim _{\alpha<\lambda} \operatorname{dir} P_{\alpha}$ if $\lambda$ is inaccessible and $P_{\lambda}=\lim _{\alpha<\lambda}$ inv $P_{\alpha}$ otherwise. If $P_{\alpha}$ has been defined for some $\alpha \leqq \kappa$ we let $\dot{Q}_{\alpha}$ be a $P_{\alpha}$ name for the trivial poset if $\alpha$ is not Mahlo and define $P_{\alpha+1}=P_{\alpha} * \dot{Q}_{\alpha}$. If $\alpha$ is Mahlo we let $\dot{Q}_{\alpha}$ be a $P_{\alpha}$ name for the iteration $Q^{\alpha}$ (defined from the first complete sequence of terms in $V^{\boldsymbol{P}_{\alpha}}$; note that $V^{\boldsymbol{P}_{\alpha}}$ is a generic extension of $L$ and therefore has a canonical well ordering) and again set $P_{\alpha+1}=P_{\alpha} * Q_{\alpha}$.

Note that for each Mahlo cardinal $\lambda \leqq \kappa, P_{\lambda}$ is $\lambda$ c.c. and at each intermediate stage $\alpha \leqq \kappa$ the iteration factors in a nice way, i.e., the tail $P_{\alpha+1, \kappa+1}$ is $<\mu$ Baire in $V^{P_{\alpha+1}}$ where $\mu$ is the least inaccessible $>\alpha$. More factoring arguments together with some standard forcing lore establish that

and we have

$$
\forall \alpha \leqq \kappa+1 \|_{P_{\alpha}} G C H,
$$

$$
\| \bar{P}_{x+1} \neg \diamond_{\kappa, R \operatorname{Reg} \kappa} .
$$

\section{Preservation of the $\Pi_{n}^{m}$ Indescribability of $\kappa$}

Assume towards a contradiction that $\Phi(\cdot)$ is a $\Pi_{n}^{m}$ formula, $\AA \in V^{P}$ and $p^{*} \in P_{x+1}$ such that

$$
p^{*} \|_{\boldsymbol{P}_{\kappa+1}} " \Phi(\AA) \text { describes } \kappa " \text {. }
$$


Pick a large $\delta$ with $\operatorname{cof}(\delta)>\kappa$ such that $V_{\delta}$ reflects enough of $Z F C$. By a Löwenheim Skolem argument we can find a transitive $M$ of size $\kappa$ such that $M^{<\kappa} \subseteq M$ and $\kappa \in M$, together with an elementary embedding $i: M \rightarrow V_{\delta}$ with crit $(i)>\kappa$ and $\AA^{M} \in M$ with $i\left(\AA^{M}\right)=\AA$. Recall the characterization of $\Pi_{n}^{m}$ indescribability in terms of elementary embeddings that is given in [H1], Theorem 1.3. Since $\kappa$ is $\Pi_{n}^{m}$ indescribable in the ground model we can find, in the ground model, an elementary embedding $j: M$ $\rightarrow N$ with critical point $\kappa$ where $N$ is transitive, $|N|=\left|V_{\kappa+m-1}\right|$, and $N$ is $\Sigma_{n-1}^{m}$ correct for $\kappa$.

We aim to find generic objects, $G^{V}, G^{M}$, and $G^{N}$ (over the indicated models for the forcing notions $P_{\kappa+1}, P_{\kappa+1}^{M}, P_{j(\kappa)+1}^{N}$ ) such that $p^{*} \in G^{V}$ and $i$ and $j$ lift, i.e., we obtain elementary embeddings if we define for $\tau \in M^{P_{\kappa+1}^{M}} i\left(\tau^{G^{M}}\right)=(i(\tau))^{G^{V}}$ and $j\left(\tau^{G^{M}}\right)=(j(\tau))^{G^{N}}$. Then we have

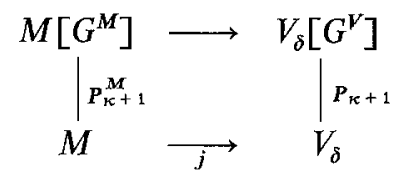

and

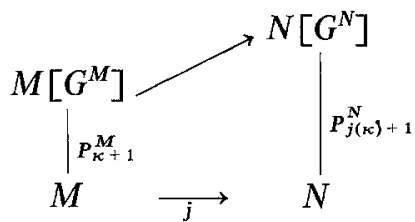

In addition we want $G^{N} \in V\left[G^{V}\right]$ and $N\left[G^{N}\right] \sum_{n-1}^{m}$ correct for $\kappa$ in $V\left[G^{V}\right]$. When all this has been achieved we arrive at a contradiction as follows: Since $p^{*} \in G^{V}$ and $\Phi$ is $\Pi_{n}^{m}$ we get by $\Sigma_{n-1}^{m}$ correctness

$$
\begin{gathered}
N\left[G^{N}\right]=" \exists \alpha<j(\kappa) V_{\alpha} \models \Phi\left(j(A) \cap V_{\alpha}\right), \\
\text { where } A=(\AA)^{G^{V}}=i\left(\left(\AA^{M}\right)^{G^{M}}\right)=\left(A^{M}\right)^{G^{M}} \in M\left[G^{M}\right] .
\end{gathered}
$$

By the elementarity of $j$ we obtain

$$
M\left[G^{M}\right] \vDash " \exists \alpha<\kappa V_{\alpha} \models \Phi\left(A \cap V_{\alpha}\right) " .
$$

We shall see that $M\left[G^{M}\right]$ is still closed under $<\kappa$ sequences in $V\left[G^{V}\right]$, and thus, in $V\left[G^{V}\right]$

$$
\exists \alpha<\kappa V_{\alpha} \models \Phi\left(A \cap V_{\alpha}\right),
$$

i.e., $\Phi(A)$ reflects - a contradiction!

We shall now describe the construction of the generic objects $G^{V}, G^{M}$, and $G^{N}$ under the assumption that $\kappa$ is $\Pi_{n}^{m}$ indescribable where $m \geqq 2$. The case $m=1$ causes an extra technical problem, and we shall deal with that at the end of the paper. Let $G_{\kappa}$ be $V$ generic for $P_{\kappa}$ with $p^{*} \mid \kappa \in G_{\kappa}$. Clearly $G_{\kappa}$ is also $M$ generic for $P_{\kappa}=P_{\kappa}^{M}$ and $N$ generic for $P_{\kappa}=P_{\kappa}^{N}$. Since $i(p)=p$ for all $p \in P_{\kappa} i$ lifts, i.e.,

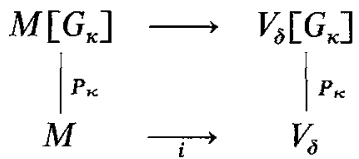


and clearly $M\left[G_{\kappa}\right]^{<\kappa} \subseteq M\left[G_{\kappa}\right]$ in $V\left[G_{\kappa}\right]$. In order to construct an $N$ generic for $P_{j(\kappa)}^{N}$ which allows us to lift $j$ we note that $j(p)=p$ for all $p \in P_{\kappa} \operatorname{since}$ crit $(j)=\kappa$. Hence with any $N\left[G_{\kappa}\right]$ generic $G_{\kappa, j(\kappa)}$ for the tail $P_{\kappa, j(\kappa)}^{N\left[G_{\kappa}\right]}$ the embedding $j$ will lift to $j: M\left[G_{k}\right] \hookrightarrow N\left[G_{k}, G_{k, f(k)}\right]$. Of course we want to pick $G_{k, j(x)}$ such that $N\left[G_{\kappa}, G_{\kappa, j(\kappa)}\right]$ remains $\sum_{n-1}^{m}$ correct for $\kappa$ in $V\left[G^{V}\right]$.

Since $\left|P_{\kappa}\right|=\kappa$ and $P_{\kappa}$ has the $\kappa$ c.c. $N\left[G_{\kappa}\right]$ is $\sum_{n-1}^{m}$ correct for $\kappa$ in $V\left[G_{\kappa}\right]$ (cf. [H1] Lemma 1.2, part 1). Moreover, ${ }^{N} Q^{\kappa}$ (the forcing that $N\left[G_{\kappa}\right]$ wants to do at stage $\kappa$ of the iteration in $N$ ) is the same as $Q^{\kappa}$ (the forcing that $V\left[G_{\kappa}\right]$ wants to do at stage $\kappa$ of the iteration in $V$ ) because $N\left[G_{\kappa}\right]$ is closed under $\kappa$ sequences in $V\left[G_{\kappa}\right]$ and in both models we take the $L\left[G_{\kappa}\right]$ first complete enumeration of nice $A d d\left(\kappa^{+}, \kappa\right)$ names for subsets of $\kappa$ and define from that the forcing for killing $\diamond_{\kappa, \text { Regn } \kappa}$.

One may now attempt to proceed as follows: Let $G$ be $V\left[G_{\kappa}\right]$ generic for ${ }^{N} Q^{\kappa}$ with $p^{*} \in G_{\kappa} * G$.

Lemma 2.1. $N\left[G_{\kappa}, G\right]$ is $\sum_{n-1}^{m}$ correct for $\kappa$ in $V\left[G_{\kappa}, G\right]$.

Proof. We show by induction on $k \leqq n-1$ that $N\left[G_{\kappa}, G\right]$ is $\Sigma_{k}^{m}$ correct for $\kappa$ in $V\left[G_{\kappa}, G\right]$. For $k=0$ note that $\left.N\left[G_{\kappa}, \bar{G}\right]^{\left|V_{\kappa+m-2}\right| \subseteq N} \subseteq G_{\kappa}, G\right]$ in $V\left[G_{\kappa}, G\right]$ since $Q^{\kappa}$ is $\kappa^{+}$c.c. in both models. Now suppose $\Phi(\cdot)$ is $\Pi_{k+1}^{m}$ with $k+1 \leqq n-1$ and $A \in\left(N\left[G_{\kappa}, G\right]\right)_{\kappa+m}$ and $N\left[G_{\kappa}, G\right] \models " V_{\kappa} \models \Phi(A)$ ". Pick a (code for a) name

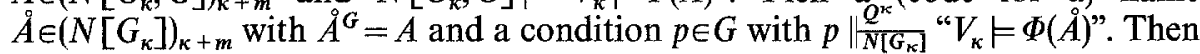
by induction hypothesis, applied in $N\left[G_{k}\right]$

$$
\begin{aligned}
& \forall \mathscr{M}\left[\mathscr{M} \text { transitive } \wedge|\mathscr{M}|=\left|V_{\kappa+m-1}\right| \wedge \mathscr{M}=Z F^{-} \wedge \mathscr{M} \Sigma_{k}^{m}\right. \text { correct for } \\
& \left.\kappa \wedge \AA \in \mathscr{M} \cdot \Rightarrow \cdot \mathscr{M} \models p \| \underline{Q^{\kappa}} \text { “ } V_{\kappa}=\Phi(\AA) ”\right] \text {. }
\end{aligned}
$$

Since this is $\Pi_{k+1}^{m}(\AA)$ it must also hold in $V\left[G_{\kappa}\right]$. By the reflection principle of $Z F$, we obtain in $V\left[G_{k}\right] p \| \underline{Q}^{\kappa}$ “ $V_{k} \models \Phi(\AA) "$. Hence $V_{k} \models \Phi(A)$ in $V\left[G_{\kappa}, G\right]$.

Recall that from the viewpoint of $N\left[G_{\kappa}, G\right]$, the tail $P_{\kappa+1, j(\kappa)}^{N\left[G_{\kappa}, G\right]}$ has for each $v<\mu$ a dense $v$ closed suborder (where $\mu$ is the least inaccessible of $N$ above $\kappa$ ). Using this together with the fact that $\left|N\left[G_{\kappa}, G\right]\right|=\kappa^{+(m-1)}$ and $N\left[G_{\kappa}, G\right]$ is closed under sequences of length $\kappa^{+(m-2)}$ we can in $V\left[G_{\kappa}, G\right]$ build an $N\left[G_{\kappa}, G\right]$ generic $H$ for the tail $P_{\kappa+1, j(\kappa)}^{N\left[G_{\kappa}, G\right]}$ in the usual way. Clearly $N\left[G_{\kappa}, G, H\right]$ is still $\sum_{n-1}^{m}$ correct for $\kappa$ in $V\left[G_{\kappa}, G\right]$ as the tail is highly Baire.

Now let $g=\left\langle\left\langle S_{\alpha}, C_{\alpha}\right\rangle: \alpha\left\langle\left(\kappa^{+}\right)^{M}\right\rangle\right.$ where $G=\left\langle\left\langle S_{\alpha}, C_{\alpha}\right\rangle: \alpha\left\langle\kappa^{+}\right\rangle\right.$, i.e., $g$ is the pullback of $G$ via $i$. Since ${ }^{M} Q^{\kappa}$ (the forcing at stage $\kappa$ of the iteration in $M$ ) is $\kappa^{+}$c.c. and $\operatorname{cpt}(i)>\kappa g$ is $M\left[G_{\kappa}\right]$ generic for ${ }^{M} Q^{\kappa}$, and if we let $G^{M}=G_{\kappa} * g$ then $i$ lifts, i.e.,

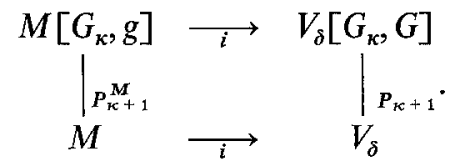

Obviously $M\left[G^{M}\right]^{<\kappa} \subseteq M\left[G^{M}\right]$ in $V\left[G^{V}\right]$ where $G^{V}=G_{\kappa} * G$.

However, this is as far as we can go. In this approach we are unable to come up with a master condition for the forcing ${ }^{N} Q^{j(\kappa)}$ at stage $j(\kappa)$ of the iteration in $N$. Since $\operatorname{cpt}(j)=\kappa$ any candidate $f$ for a master condition must have $\operatorname{supp}(f) \supseteqq\left\{\langle j(\alpha), i\rangle: \alpha<\left(\kappa^{+}\right)^{M}, i<2\right\}$ and for all $\alpha<\left(\kappa^{+}\right)^{M} f(\langle j(\alpha), 0\rangle)$ must extend $S_{\alpha}$ and $f(\langle j(\alpha), 1\rangle)$ must extend $C_{\alpha} \cup\{\kappa\}$. But $\kappa$ is regular and we cannot argue that the $\kappa$-th set in the potential diamond sequence whose name appears at coordinate $j(\alpha)$ of ${ }^{N} Q^{j(\kappa)}$ is forced to be $\neq S_{\alpha}$.

For this reason we cannot obtain the $M\left[G_{\kappa}\right]$ generic for ${ }^{M} Q^{\kappa}$ by restricting the $V\left[G_{\kappa}\right]$ generic for $Q^{\kappa}$ to its first $\left(\kappa^{+}\right)^{M}$ many coordinates. Instead, we shall work in 
$V\left[G_{\kappa}\right]$ and define a sequence $\left\langle\alpha_{r}: \zeta\left\langle\left(\kappa^{+}\right)^{M}\right\rangle\right.$ of coordinates $<\kappa^{+}$such that (with Boolean value 1 in $\left.\left(V\left[G_{\kappa}\right]\right)^{Q^{\kappa}}\right)$ the embedding $j$ will lift if we take as the $M\left[G_{\kappa}\right]$ generic for ${ }^{M} Q^{\kappa}$ the restriction of the $V\left[G_{\kappa}\right]$ generic for $Q^{\kappa}$ to the coordinates $\left\langle\left\langle\alpha_{\zeta}, i\right\rangle: \zeta<\left(\kappa^{+}\right)^{M}, i<2\right\rangle$.

Denote by $\left\langle\tau_{\alpha}: \alpha<\kappa^{+}\right\rangle$the complete enumeration of nice $\operatorname{Add}\left(\kappa^{+} \times 2, \kappa\right)$ names for subsets of $\kappa$ that we use to define $Q^{\kappa}$ in $N\left[G_{\kappa}\right]$ and $V\left[G_{\kappa}\right]$. Denote by $\left\langle\tau_{\alpha}^{N\left[G_{\kappa}, G, H\right]}: \alpha<j(\kappa)^{+}\right\rangle$the complete enumeration of nice $\operatorname{Add}\left(j\left(\kappa^{+}\right) \times 2, j(\kappa)\right)$ names for subsets of $j(\kappa)$ that we use to define $Q^{j(\kappa)}$ (the $j(\kappa)$-th stage of the iteration in $\left.N\right)$. By induction on $\zeta<\kappa^{+}$we shall now define sequences

$$
\left\langle\alpha_{\zeta}: \zeta\left\langle\left(\kappa^{+}\right)^{M}\right\rangle\right.
$$

and

$$
\left\langle f_{\zeta}: \zeta<\left(\kappa^{+}\right)^{M}\right\rangle
$$

in $V\left[G_{\kappa}\right]$. Suppose $\zeta<\left(\kappa^{+}\right)^{M}$ and $\left\langle\alpha_{\eta}: \eta\langle\zeta\rangle\right.$ and $\left\langle f_{\eta}: \eta\langle\zeta\rangle\right.$ have been defined such that for all $\eta<\zeta$

(i) $\alpha_{\eta}<\kappa^{+}$

(ii) $\tau_{\alpha_{\eta}}=\left(\hat{\tau}_{\eta}\right)^{\pi_{\eta}}$ (where $\pi_{\eta}=\left\{\left\langle v, \alpha_{v}\right\rangle: v<\eta\right\}$, and where $\left(\hat{\tau}_{\eta}\right)^{\pi_{\eta}}$ denotes the shift of $\hat{\tau}_{\eta}$ according to $\pi_{\eta}$ ).

(iii) $\| \frac{Q^{\kappa}}{V\left[G_{\kappa}\right]}$ “ $f_{\eta} \in \widetilde{Q}_{j(\eta)+1}^{j(\kappa)}$ (as computed in $\left.N\left[G_{\kappa}, \stackrel{\circ}{G}, \stackrel{\circ}{H}\right]\right) \wedge f_{\eta}(\langle j(\eta), 0\rangle) \supseteqq 2 S_{a_{\eta}} \wedge$ $f_{\eta}(\langle j(\eta), 1\rangle) \supseteqq C_{\alpha_{\eta}} \cup\{\kappa\} ”$.

Here $\hat{G}$ denotes the canonical name $\in V^{Q^{\kappa}}$ for the generic object and $\dot{S}_{\alpha_{\eta}}\left(\AA_{\alpha_{\eta}}\right.$ resp.) is a canonical name for the subset of $\kappa$ that $\stackrel{\circ}{G}$ adds at coordinate $\left\langle\alpha_{p}, 0\right\rangle\left\langle\left\langle\alpha_{\eta}, 1\right\rangle\right.$

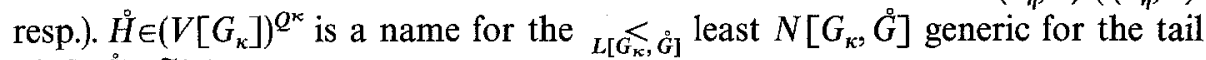
$P_{\kappa+1, j(\kappa)}^{N\left[G_{\kappa}, \mathscr{G}^{]}\right]}, \widetilde{Q}_{\alpha}^{j(\kappa)}$ (for $\left.\alpha<j(\kappa)^{+}\right)$is a $Q^{\kappa}$ name for the dense $\left(\kappa^{+}\right)^{M}$ closed suborder of $Q_{\alpha}^{j(\kappa)}$ (as computed in $\left.N\left[G_{\kappa}, \dot{G}, \dot{H}\right]\right)$.

(iv) $\forall v \leqq v^{\prime} \leqq \eta \| \frac{Q^{\kappa}}{v\left[G_{k}\right]} f_{v^{\prime}} \leqq f_{v}$.

In order to find $\alpha_{\zeta}$ and $f_{\zeta}$ we proceed as follows. For $\gamma<\kappa^{+}$let $b_{\gamma}=\left\{p \in Q^{\kappa}: p \| \frac{Q^{\kappa}}{V\left[G_{\kappa}\right]}\right.$ “ $\gamma<\kappa^{+}$is minimal with the property that in

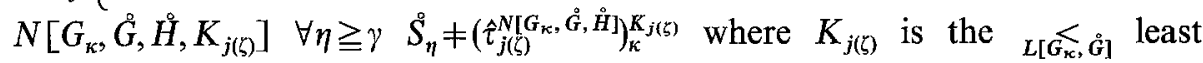
$N\left[G_{\kappa}, \stackrel{\circ}{G}, \stackrel{\circ}{H}\right]$ generic for $Q_{j(\zeta)}^{j(\kappa)}$ that extends $f_{v}$ for all $\left.v<\zeta ”\right\}$.

To justify the definition of $b_{\gamma}$ note that $\left(\hat{t}_{j(\zeta)}^{N\left[G_{k}, \dot{G}, \dot{H}\right]}\right)_{k}^{K_{j(\zeta)}}$, being a subset of $\kappa$, is actually an element of $N\left[G_{\kappa}\right.$, some sufficiently long initial segment of $\left.G\right]$ since both $Q^{j(\kappa)}$ and the tail $P_{\kappa+1, j(\kappa)}$ are $\kappa$ Baire and $Q^{\kappa}$ is $\kappa^{+}$c.c. By the $\kappa^{+}$c.c. of $Q^{\kappa}$ again, $\left|\left\{\gamma<\kappa^{+}: b_{\gamma} \neq \emptyset\right\}\right| \leq \kappa$. Now let $\alpha_{\zeta}$ be the least ordinal $<\kappa^{+}$such that $\alpha_{\zeta}>\sup \left\{\gamma<\kappa^{+}: b_{y} \neq \emptyset\right\} \cup \sup \left\{\alpha_{\eta}: \eta<\zeta\right\}$ and $\tau_{\alpha_{\zeta}}=\left(\hat{t}_{\zeta}\right)^{\pi_{\zeta}}\left(\right.$ where $\left.\pi_{\zeta}=\left\{\left\langle\eta, \alpha_{\eta}\right\rangle: \eta<\zeta\right\}\right)$. It follows that

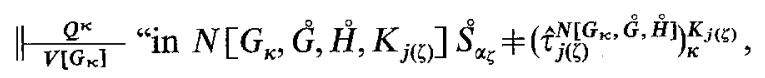


where $K_{j(5)}$ is as above". Thus we can find $f \in\left(V\left[G_{\kappa}\right]\right)^{Q^{\kappa}}$ such that

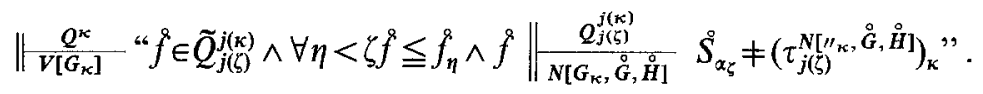

Let $f^{*}$ be the ${ }_{L\left[G_{\kappa}\right]}$ least such $f$ and pick $f_{\zeta} \in V\left[G_{\kappa}\right]^{Q_{\kappa}}{ }_{L\left[\xi_{\kappa]}\right.}$ least such that

$$
\| \frac{Q^{\kappa}}{V\left[G_{x]}\right]} “ f_{\zeta} \in \widetilde{Q}_{j(\zeta)+1}^{j(\kappa)} \wedge f_{\zeta} \leqq f^{*} \wedge f_{\zeta}(\langle j(\zeta), 0\rangle) \supseteqq S_{\alpha_{\zeta}} \wedge f_{\zeta}(\langle j(\zeta), 1\rangle) \supseteqq \check{C}_{\alpha_{\zeta}} \cup\{\kappa\} ” .
$$

Recall that $\pi_{\zeta}$ induces an isomorphism of $Q_{\zeta}^{\kappa}$ with $\left\{\left\langle\alpha_{\eta}, i\right\rangle: \eta<\left(\kappa^{+}\right)^{M, i<2\}} Q_{\alpha_{\zeta}}^{\kappa}\right.$ which is a complete suborder of $Q_{\alpha \zeta}$ since $\left\{\left\langle\alpha_{\eta}, i\right\rangle: \eta<\zeta, i<2\right\} \subseteq \kappa^{+} \times 2$ is a complete set of coordinates. Obviously (i)-(iv) hold with $\alpha_{\underline{\zeta}}$ and $f_{\xi}$.

Now let $G$ be $Q^{\kappa}$ generic over $V\left[G_{\kappa}\right]$. Let $H$ be the ${ }_{L\left[G_{\kappa}, G\right]}^{\leftarrow}$ least $N\left[G_{\kappa}, G\right]$ generic for the tail $P_{\kappa+1}^{N\left[G_{\kappa}, G\right]}$. Since $\widetilde{Q}^{j(\kappa)}$ is $\left(\kappa^{+}\right)^{M}$ closed there is a condition $q$ extending $f_{\zeta}^{G}$ for all $\zeta<\left(\kappa^{\star}\right)^{M}$. By our construction $q$ is a master condition. In $V\left[G_{\kappa}, G\right]$ build a generic $K$ containing $q$ for the forcing $Q^{j(\kappa)}$ over $N\left[G_{\kappa}, G, H\right]$ by using that $Q^{j(\kappa)}$ has a $\kappa^{+(m-2)}$ closed dense suborder and $\left|N\left[G_{\kappa}, G, H\right]\right|=\kappa^{+(m-1)}$. Notice that $\left\{\left\langle\alpha_{\zeta}, i\right\rangle: \zeta<\left(\kappa^{+}\right)^{M}, i<2\right\} Q^{\kappa}$ is a complete suborder of $Q^{\kappa}$ which is isomorphic to $Q_{\left(\kappa^{+}\right)^{m}}^{\kappa}$ via the map induced by $\bigcup_{\zeta<\left(\kappa^{+}\right)^{M}} \pi_{\zeta}$. Let $g$ be the transform under this isomorphism of the restriction of $G$ to the coordinates $\left\{\left\langle\alpha_{\xi}, i\right\rangle: \zeta<\left(\kappa^{+}\right)^{M}, i<2\right\}$. Since crit $\left.(i)\right\rangle \kappa$ and $M\left[G_{\kappa}\right]$ is closed under $<\kappa$ sequences, $Q_{\left(\kappa^{+}\right)^{M}}^{\kappa^{*}}$ is (essentially) equal to the forcing at stage $\kappa$ of the iteration in $M$. Certainly $g$ is $M\left[G_{\kappa}\right]$ generic for $Q_{\left(\kappa^{+}\right)^{M}}^{\kappa}$. Moreover, since $q \in K$ the embedding $j$ lifts, i.e.,

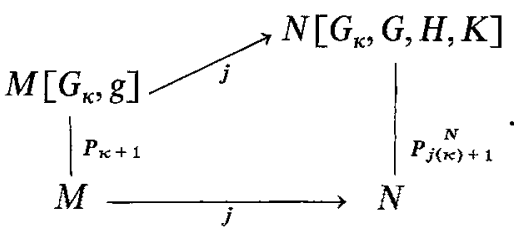

By Baireness $N\left[G_{\kappa}, G, H, K\right]$ is still $\sum_{n-1}^{m}$ correct for $\kappa$ in $V\left[G_{\kappa}, G\right]$. Notice that we can extend $\bigcup_{\zeta<\left(\kappa^{+}\right)^{M}} \pi_{\zeta}$ to a bijection on $\kappa^{+}$which gives an automorphism of $Q^{\kappa}$ as in the proof of 1.4. Let $G^{*}$ be the pointwise preimage of $G$ under this automorphism. Since $\left\langle\alpha_{\zeta}: \zeta<\left(\kappa^{+}\right)^{M}\right\rangle$ was defined in $V\left[G_{\kappa}\right] G^{*}$ is $V\left[G_{\kappa}\right]$ generic for $Q^{\kappa}$ and $V\left[G_{\kappa}, G\right]=V\left[G_{\kappa}, G^{*}\right]$. Since $g$ is an initial segment of $G^{*}, i$ also lifts as crit $(i)>\kappa$, i.e.,

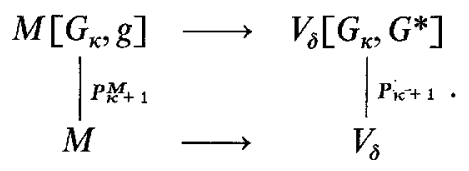

Moreover, $M\left[G_{\kappa}, g\right]^{<\kappa} \leqq M\left[G_{\kappa}, g\right]$ in $V\left[G_{\kappa}, G^{*}\right]$ since the forcing is $<\kappa$ Baire. Finally note that we clearly could have picked $G$ to ensure that $\left(p^{*}(\kappa)\right)^{G_{\kappa}} \in G^{*}$. Thus set $G_{\kappa} * G^{*}=G^{V}, G_{\kappa} * g=G^{M}$, and $G_{\kappa} * G^{*} * H * K=G^{N}$.

This finishes the proof for the $\Pi_{n}^{m}$ case where $m \geqq 2$. For the $\Pi_{n}^{1}(n \geqq 1)$ case the above proof has to be modified. If $N$ and its generic extensions (in the notation from above) are only $\Sigma_{n-1}^{1}$ correct for $\kappa$ in the appropriate generic extensions of $V$, 
we cannot carry out the construction of the master condition in the last step for lifting the embedding $j$. That argument relied on the closure of the generic extension of $N$ under $\kappa$ sequences in the generic extension of $V$ whereas now we only have closure under $<\kappa$ sequences available.

In order to modify the argument we start by choosing a $\Pi_{n}^{1}$ formula $\Phi$ together with $\AA, p^{*}, \delta, M, \AA^{M}$, and $i$ as before. Since $\kappa$ is $\Pi_{n}^{1}$ indescribable we can find some transitive $N$ of size $\kappa$ which is $\Sigma_{n-1}^{\mathbf{1}}$ correct for $\kappa$ and an embedding $j: M \rightarrow N$ with $\operatorname{cpt}(j)=\kappa$. An inspection of the proof of Theorem 1.3 in [H1] yields that we can get such $N$ and $j$ with $M, j \in N$ and $|M|^{N}=\kappa$. Standard arguments enable us to build $\tilde{j}$ and $\tilde{N}$, both elements of $N$, such that $\tilde{j}: M \underset{\Sigma_{k}}{\longrightarrow} \tilde{N}$ (where the integer $k$ is chosen large enough for the argument that follows), crit $(\tilde{j})=\kappa$ and $N \models "|\tilde{N}|$ $=\kappa^{+} \wedge \tilde{N}^{\kappa} \subseteq \tilde{N}^{\prime \prime}$.

Then we choose a $V$ generic $G_{\kappa}$ for $P_{\kappa}$ with $p^{*} \mid \kappa \in G_{\kappa}$. Note that $P_{\kappa}^{M}=P_{\kappa}^{\tilde{N}}$ $=P_{\kappa}^{N}=P_{\kappa^{*}}$. Thus $G_{\kappa}$ is also $P_{\kappa}$ generic over $M, \tilde{N}$, and $N$. Moreover, $M\left[G_{\kappa}\right]^{<\kappa} \subseteq M\left[G_{\kappa}\right]$ in $V\left[G_{\kappa}\right], \tilde{N}\left[G_{\kappa}\right]^{\kappa} \subseteq \tilde{N}\left[G_{\kappa}\right]$ in $N\left[G_{\kappa}\right]$ which is $\Sigma_{n-1}^{\mathbf{1}}$ correct for $\kappa$ in $V\left[G_{\kappa}\right]$, and $i$ lifts, i.e.,

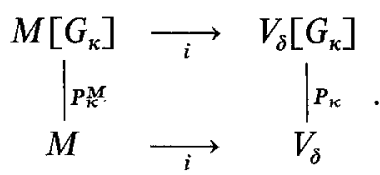

Let $\left\langle{ }^{M} \tau_{\alpha}: \alpha<\left(\kappa^{+}\right)^{M}\right\rangle$ be the enumeration in $M\left[G_{\kappa}\right]$ that we use to define ${ }^{M} Q^{\kappa}$ (stage $\kappa$ of $P_{\kappa+1}^{M}$. Extend $\left\langle{ }^{M} \tau_{\alpha}: \alpha\left\langle\left(\kappa^{+}\right)^{M}\right\rangle\right.$ to a complete enumeration, say $\left\langle{ }^{N} \tilde{\tau}_{\alpha}: \alpha<\left(\kappa^{+}\right)^{N}\right\rangle$ in $\tilde{N}\left[G_{\kappa}\right]$ and let ${ }^{N} \widetilde{Q}^{\kappa}$ be the corresponding poset for killing $\diamond_{\kappa, \text { Regn } \kappa}$ in $\tilde{N}\left[G_{\kappa}\right]$ (and hence also in $N\left[G_{\kappa}\right]$ ). Finally extend $\left\langle{ }^{N} \tilde{\tau}_{\alpha}: \alpha\left\langle\left(\kappa^{+}\right)^{N}\right\rangle\right.$ to a complete enumeration, say $\left\langle\tilde{\tau}_{\alpha}: \alpha<\kappa^{+}\right\rangle$in $V\left[G_{\kappa}\right]$ and denote by $\widetilde{Q}^{\kappa}$ the corresponding poset. Working in $N\left[G_{\kappa}\right]$, and using ${ }^{N} \widetilde{Q}^{\kappa}$ instead of ${ }^{N} Q^{\kappa}$ (which is the poset at stage $\kappa$ of the iteration in $N$ ) we can now repeat the old argument to define a sequence $\left\langle\left\langle\alpha_{\zeta}, i\right\rangle: \zeta\left\langle\left(\kappa^{+}\right)^{M}, i<2\right\rangle\right.$ of coordinates $\in\left(\kappa^{+}\right)^{N} \times 2$ such that with boolean value 1 (as computed in $\left(N\left[G_{k}\right]\right)^{N} \tilde{Q}^{\kappa}$ ) the embedding $\tilde{j}$ can be lifted if we take as the $M\left[G_{\kappa}\right]$ generic object for ${ }^{M} Q^{\kappa}$ the restriction of the $N\left[G_{\kappa}\right]$ generic object for ${ }^{N} \widetilde{Q}^{\kappa}$ to the coordinates $\left\langle\left\langle\alpha_{\zeta}, i\right\rangle: \zeta<\left(\kappa^{+}\right)^{M}, i<2\right\rangle$.

Now pick a $V\left[G_{k}\right]$ generic $G$ for $Q^{\kappa}$. By 1.4 there is a $\widetilde{Q}^{\kappa}$ generic $\tilde{G}$ such that $V\left[G_{\kappa}, G\right]=V\left[G_{\kappa}, \widetilde{G}\right]$. Let ${ }^{N} \widetilde{G}$ be the restriction of $\widetilde{G}$ to the first $\left(\kappa^{+}\right)^{N}$ many coordinates. Clearly ${ }^{N} \widetilde{G}$ is ${ }^{N} \widetilde{Q}^{\kappa}$ generic over $N\left[G_{\kappa}\right]$. Let $g$ denote the restriction of ${ }^{N} \tilde{G}$ to the coordinates $\left\langle\left\langle\alpha_{\xi}, i\right\rangle: \xi\left\langle\left(\kappa^{+}\right)^{M}, i<2\right\rangle\right.$. We know that $g$ is $M\left[G_{\kappa}\right]$ generic for ${ }^{M} Q^{\kappa}$ and that within $N\left[G_{\kappa},{ }^{N} \widetilde{G}\right]$ we can build generics $H$ and $K$ such that $\tilde{j}$ lifts to $\tilde{j}: M\left[G_{\kappa}, g\right] \underset{\Sigma_{k}}{\longrightarrow} \tilde{N}\left[G_{\kappa},{ }^{N} \widetilde{G}, H, K\right]$. Since $\left\langle\left\langle\alpha_{\zeta}, i\right\rangle: \zeta\left\langle\left(\kappa^{+}\right)^{M}\right.\right.$, $i<2\rangle \in N\left[G_{\kappa}\right] \subseteq V\left[G_{\kappa}\right] V\left[G_{\kappa}, G\right] \stackrel{=}{=}\left[G_{\kappa}, G^{*}\right]$ where as above $G^{*}$ denotes the generic that is obtained from $\tilde{G}$ by the automorphism of $\widetilde{Q}^{\kappa}$ induced by extending the map $\zeta \rightarrow \alpha_{\zeta}\left(\zeta<\left(\kappa^{+}\right)^{M}\right)$ to a bijection of $\kappa^{+}$as in the proof of 1.4. Moreover, $i$ can be lifted to an embedding $i: V\left[G_{\kappa}, g\right] \rightarrow V_{\delta}\left[G_{\kappa}, G^{*}\right]$ and if we let $A_{\mathscr{Q} f}(\AA)^{G_{\kappa}^{*} G^{*}}$ then $A=i\left(\left(\AA^{M}\right)^{G_{\kappa} * g}\right)=\left(\AA^{M}\right)^{G_{\kappa^{*} g}} \in M\left[G_{\kappa}, g\right]$. Clearly, we could have picked $G$ such that $\left(p^{*}(\kappa)\right)^{G_{\kappa}} \in G^{*}$. Note also that $\tilde{N}\left[G_{\kappa},{ }^{N} \tilde{G}, H, K\right]$ is closed under $\kappa$ sequences inside $N\left[G_{\kappa},{ }^{N} \widetilde{G}\right]$ and $N\left[G_{\kappa},{ }^{N} \widetilde{G}\right]$ is still $\Sigma_{n-1}^{1}$ correct for $\kappa$ in $V\left[G_{\kappa}, \widetilde{G}\right]$ (for this use the method from the proof of 2.1 together with a factoring argument as 
in the proof of 3.3.1 in [H2]). Thus, as $p^{*} \in G_{\kappa} * G^{*}$ and $\tilde{N}\left[G_{\kappa},{ }^{N} \widetilde{G}, H, K\right]$ is $\Sigma_{n-1}^{1}$ correct for $\kappa$ in $V\left[G_{\kappa}, G^{*}\right]$

$$
\tilde{N}\left[G_{\kappa},{ }^{N} \widetilde{G}, H, K\right] \models \exists \alpha<\tilde{j}(\kappa) V_{\alpha} \models \Phi\left(\tilde{j}(A) \cap V_{\alpha}\right) .
$$

By the elementarity of $\tilde{j}$ we obtain

$$
M\left[G_{\kappa}, g\right] \models \exists \alpha<\kappa V_{\alpha} \models \Phi\left(A \cap V_{\alpha}\right) .
$$

But clearly $M\left[G_{\kappa}, g\right]^{<\kappa} \cong M\left[G_{\kappa}, g\right]$ in $V\left[G_{\kappa}, G^{*}\right]$. So $\Phi(A)$ reflects in $V\left[G_{\kappa}, G^{*}\right]-$ a contradiction.

\section{Concluding Remarks}

The natural question to ask is whether this proof can be modified in order to get $\neg \diamond_{\kappa, \kappa}$ at a $\Pi_{n}^{m}$ indescribable cardinal $\kappa$ in the generic extension. However, Jensen [J] has recently shown that the non-existence of $0^{\#}$ implies $\diamond_{\kappa, E_{\omega} \cap \kappa}$ for any Mahlo cardinal $\kappa$. (Here and below $E_{\omega}$ denotes the class of all cardinals of cofinality $\omega$ ). Thus it seems one has to assume stronger hypotheses in the ground model and then use a different forcing altogether in order to make such weak versions of $\diamond$ fail.

Woodin (cf. [CW]) starts with a sufficiently hypermeasurable cardinal $\kappa$ at which $G C H$ fails badly enough and uses Radin forcing to produce a model where $\kappa$ is greatly Mahlo and $\diamond_{\kappa, \kappa}$ fails (in fact no sequence in the generic extension guesses every subset in the ground model stationary often). It is not known how to preserve the weak compactness of $\kappa$.

Question 1.Can $\diamond_{\kappa, \kappa}$ fail at a weakly compact cardinal $\kappa$ (or more general for $\kappa \Pi_{n}^{m}$ indescribable)?

Gregory [G] has shown that (in $Z F C$ ) $\diamond_{\alpha_{2}, E_{\omega} \cap \alpha_{2}}$ follows from $C H+2^{\alpha_{1}}=\alpha_{2}$. Certain aspects of the proof of this fact lead to:

Question 2. Does $G C H$ on a stationary set of singulars below $\kappa$ imply $\diamond_{\kappa, \kappa}$ when $\kappa$ is inaccessible?

Alternatively we may ask

Question 3. What is the consistency strength of $\neg \diamond_{\kappa, \kappa}$ at an inaccessible $\kappa$ ?

Recall that $\diamond_{\kappa, \kappa}$ implies that $N S_{\kappa}$ (the non-stationary ideal on $\kappa$ ) is not $2^{\kappa}$ saturated.

Question 4. Is it possible to have an inaccessible cardinal $\kappa$ such that $N S_{\kappa} \mid E_{\omega}$ is $2^{\kappa}$ saturated?

Looking at the other end of the large cardinal scale, one can consider

Question 5. $\operatorname{CON}\left(Z F C+\exists \kappa\left(\kappa\right.\right.$ huge $\left.\left.+\neg \diamond_{\kappa, E_{\omega} \cap \kappa}\right)\right)$ ?

There is no hope of being able to give a positive answer to this question by using Radin forcing. For by a theorem of Solovay [So], if $\kappa$ is huge then there is an $\omega$-closed unbounded class of cardinals below $\kappa$ at which $G H C$ holds. In Woodin's model above, for example, $G C H$ fails at $\kappa$ (which can be fixed by adding a Cohen subset of $\kappa^{+}-$this will not resurrect $\diamond_{\kappa, \kappa}$ ) but also at "many" $\lambda<\kappa$. More problems arise from the fact that, by a result of Shelah [Sh], if $\kappa$ is huge the 
combinatorial principle ${ }_{w} \square_{\delta}$ (which states that there is a sequence $\left\langle C_{\alpha}: \alpha<\delta^{+}\right\rangle$of club set $C_{\alpha} \subseteq \alpha$ with the property that each $C_{\alpha}$ has order type $=\operatorname{cof}(\alpha)$ and $\left.\forall \beta<\delta^{+}\left|\left\{C_{\alpha} \cap \beta: \alpha<\delta^{+}\right\}\right| \leqq \delta\right)$ fails at all sufficiently large strong limits of cofinality $\omega$ below $\kappa$. On the other hand, in the Radin approach we add "many" ${ }_{w} \square_{\delta}$ sequences when we change the cofinality of $\delta$ (where $\delta^{<\delta}=\delta$ ) to $\omega$. For these reasons any forcing that gives a positive answer to Question 5 has to be of a rather pathological nature.

\section{References}

[CW] Cummings, J., Woodin, H.: Generalized prikry forcing.

[D1] Devlin, K.J.: The axiom of constructibility. Lect. Notes Math. 617, VIII $+95(1977)$

[D2] Devlin, K.J.: Constructibility. Berlin Heidelberg New York: Springer 1984

[G] Gregory, J.: Higher soushin trees and the generalized continuum hypothesis. J. Symb. Logic 41, 663-671 (1976)

[H1] Hauser, K.: Indescribable cardinals and elementary embeddings. J. Symb. Logic 56, $439457(1991)$

[H2] Hauser, K.: The indescribability of the order of the indescribable cardinals. Ann. Pure Appl. Logic 57 (1), 45-91 (1992)

[J] Jensen, R.B.: Lecture at the set theory meeting at Oberwolfach. November 1991

[KM] Kanamori, A., Magidor, M.: The evolution of large cardinal axioms in set theory. In: Müller, G.H., Scott, D.S. (eds.). Higher set theory. Proc. Oberwolfach 1977. (Lect. Notes Math., vol. 669, pp. 99-275). Berlin Heidelberg New York: Springer 1978

[Sh] Shelah, S.: On the successors of singular cardinals. In: Boffa, M., van Dalen, D., McAloon, K. (eds.) Logic colloquium 78, vol. 97, pp. 357-380. Amsterdam: NorthHolland 1979

[So] Solovay, R.M.: Strongly compact cardinals and the GCH. Tarski symposium. Proc. Symp. Pure Math. AMS, Providence, RI 25, 365-372 (1974) 